To appear in the International Journal of Production Research

Vol. 00, No. 00, 00 Month 20XX, 1-18

\title{
Analysis of irregular three-dimensional packing problems in Additive Manufacturing: A new taxonomy and dataset
}

\author{
Luiz J.P. Araújo ${ }^{a *}$, Ender Özcan ${ }^{\mathrm{a}}$, Jason A.D. Atkin ${ }^{\mathrm{a}}$ and Martin Baumers ${ }^{\mathrm{b}}$ \\ ${ }^{a}$ Automated Scheduling, Optimisation and Planning Group School of Computer Science, University of \\ Nottingham, Nottingham NG8 1BB, UK; ${ }^{b}$ Centre for Additive Manufacturing, Faculty of Engineering, \\ University of Nottingham, Nottingham NG7 2RD, UK
}

( $v 5.0$ released June 2015$)$

\begin{abstract}
With most Additive Manufacturing (AM) technology variants, build processes take place inside an internal enclosed build container, referred to as a 'build volume'. It has been demonstrated that the effectiveness with which this volume is filled with product geometries forms an important determinant of overall process efficiency in AM. For effective operations management, it is important to understand not only the problem faced, but also which methods have proved effective (or ineffective) for problems with these characteristics in the past. This research aims to facilitate this increased understanding. The build volume packing task can be formulated as a three-dimensional irregular packing (3DIP) problem, which is a combinatorial optimisation problem requiring the configuration of a set of arbitrary volumetric items. This paper reviews existing general cutting and packing taxonomies and provides a new specification which is more appropriate for classifying the problems encountered in AM. This comprises a clear-cut problem definition, a set of precise categorisation criteria for objectives and problem instances, and a simple notation. Furthermore, the paper establishes an improved terminology with terms that are familiar to, but not limited to, researchers and practitioners in the field of AM. Finally, this paper describes a new dataset to be used in the evaluation of existing and proposed computational solution methods for 3DIP problems encountered in AM and discusses the importance of this research for further underpinning work.
\end{abstract}

Keywords: packing problems; benchmarking; additive manufacturing; 3D printing; classification

\section{Introduction}

Additive manufacturing (AM), also known as 3D printing, refers to a set of technologies that allow the production of highly complex parts in an incremental layer-by-layer material deposition process. With most AM technology variants, this process takes place inside the machine in an enclosed build container, referred to as a 'build volume'. One defining characteristic of AM technology is that it does not employ any dedicated physical tooling such as moulds, cutting implements or dies (Hague, Mansour, and Saleh 2004; Gibson, Rosen, and Stucker 2014). This aspect gives AM the capability to manufacture components that would be very difficult, or impossible, to produce by conventional manufacturing processes such as injection moulding or computer numerically controlled machining.

By opening up previously unavailable regions of design space (de Mul 2016), products that were conventionally designed and manufactured as assemblies of multiple components can now be joined to form consolidated designs to be manufactured as single items (Hague, Campbell, and Dickens 2003). Additionally, the absence of physical tooling allows the cost efficient manufacturing of parts in small volumes, down to a single unit. This allows the production of highly customised or differentiated products (Tuck et al. 2008) and the adoption of innovative supply chains (Achillas,

*Corresponding author. Email: psxlja@exmail.nottingham.ac.uk 
Tzetzis, and Raimondo 2017).

AM was initially developed in the 1980s to prototype physical models that would provide insights into a product before committing to final manufacturing processes (Jacobs 1992). However, in recent years AM has been adopted for the manufacture of end-use products, for example in the aerospace, industrial equipment, automotive and medical domains. AM carries significant potential for adoption in applications that are characterised by high per-unit revenues and that benefit from high degrees of geometric complexity or customisation (Tuck et al. 2008).

As a parallel manufacturing process, AM enables the contemporaneous manufacture of different part geometries in a single build volume (Ruffo and Hague 2007). This gives rise to a build volume packing problem arising during the machine setup process, which should be addressed through a computational solution (Nyaluke et al. 1996; Hur et al. 2001). Baumers et al. (2013, 2017) have shown that the effectiveness of addressing this problem in AM execution forms an important determinant of manufacturing cost.

It has been argued that AM, particularly with respect to the absence of physical tooling, should be seen as a flexible manufacturing system (Weller, Kleer, and Piller 2015). Fixed costs for AM, for example from process warm up and cool down, resemble other setup effects identified in the operations management (OM) literature (Vokurka and O'Leary-Kelly 2000). Therefore, the actual build time for a volume will depend upon the build height and the number of build jobs needed to manufacture a set of items, which will both be impacted by the quality of the packing (Baumers et al. 2013).

There are a variety of packing approaches; the choice of the most suitable technique often depends on the characteristics of the problem, the technology employed, the material used and the production constraints (Ikonen et al. 1997; Hur et al. 2001; Canellidis et al. 2006). Some algorithms, for example, address the free arrangement of geometries within the build volume, which may result in configurations in which one item is located on top of others. Such methods are suitable for relatively unconstrained techniques such as Laser Sintering, where support structures are not needed (Gibson, Rosen, and Stucker 2014). Other technology variants require adjustments to allow for support structures such as resin vat processes or metallic powder bed processes.

Within the field of operations research (OR), there has been considerable research into cutting and packing $(\mathrm{C} \& \mathrm{P})$ algorithms. Three-dimensional irregular packing (3DIP) problems are combinatorial optimisation problems where a set of arbitrary volumetric items must be placed into given containers, or build volumes, in such a way that the total empty space (between the items) is minimised (Wäscher, Haußner, and Schumann 2007). Such optimisation problems are classified as NP-Hard (Garey and Johnson 1979). A factor that additionally increases their inherent difficulty is the presence of non-overlapping constraints between items and the edges of the container. Further variants of the problem consider other constraints, including weight distribution (Davies and Bischoff 1999), cargo stability (Hemminki, Leipala, and Nevalainen 1998), limited load bearing strength (Bischoff 2006) and packing into a non-rectangular container (Eisenbrand et al. 2005; Scheithauer, Stoyan, and Romanova 2005; Boccia et al. 2011).

In addition to overcoming the computational challenge inherent to 3DIP problems, a number of ancillary issues must be addressed when proposing practical solutions for AM. These include, but are not limited to, the following:

- Identification of the problem class, based on the existing taxonomies from the scientific literature and the technological requirements of a particular AM system. This identification will normally require relating the investigated problem to precedents from the OR literature, which contains proposed methods for efficient solutions.

- Statement of which characteristics and features of 3DIP problems are the most pertinent to the construction and tailoring of solution algorithms.

These issues are recurring and, if not met adequately, are liable to lead to a solution for the 
wrong problem or the choice of a poorly performing algorithm for a particular instance. The primary contribution of this paper is, therefore, to extend the existing general cutting and packing taxonomies (Dyckhoff 1990; Wäscher, Haußner, and Schumann 2007) by providing a clear-cut problem definition, precise categorization criteria for the objectives and problem instances, a simple yet useful notation, and establishing a terminology with terms that are familiar to, but not limited to, researchers in the field of AM. Additionally, this work provides a survey of the main AM packing problems published in the OR field, summarises solution techniques usually applicable to this context, and outlines problem features that are related to the chosen methods.

Benchmarks form a central element in assessing the performance of solutions to computational problems in-context. However, there are currently few relevant benchmarks available for 3DIP. For example, neither the EURO Special Interest Group on Cutting and Packing ${ }^{1}$ nor the ORLibrary $^{2}$ provided by Beasley (1990) contain instances of 3DIP problems. Those that do exist are not sufficiently well aligned with real-world AM cases, which poses a barrier to research in this area (Egeblad, Nielsen, and Brazil 2009). In support of the development of packing solutions and enabling their performance comparison, this paper assembles a new and more realistic 3DIP benchmark with an enriched set of features.

The remainder of this paper is organised as follows: Section 2 outlines the existing research for cutting and packing problems and introduces an extended taxonomy. This extension is formulated on the basis of a review of categorisation criteria for packing problems and classification for 3DIP problem instances encountered in AM. Section 3 surveys existing datasets and their features, it also introduces the new dataset covering a wide variety of features and contains instances that more closely simulate real-world scenarios than existing datasets. In Section 4, we discuss the outcomes of our analysis and identify the linkages between our analysis and the operations of AM technology. We conclude our paper in Section 5 with implications and suggestions for further research.

\section{Taxonomies for cutting and packing problems}

This section reviews the existing taxonomies for cutting and packing problems, the classification criteria for part geometries, and the main approaches to 3DIP problems. Moreover, it presents typical constraints and objectives for 3DIP faced in AM applications.

\subsection{Review of existing taxonomies for CESP problems}

The first typology for C\&P problems was presented by Dyckhoff (1990), who proposed representing problems based on a four-tuple: $\alpha / \beta / \gamma / \delta$. In this notation, the encountered problems are categorised according to their dimensionality $(\alpha)$, the kind of assignment $(\beta)$ or requirements regarding the selection of items and containers, the quantity and shape of containers $(\gamma)$, and the multiplicity of types of objects demanded $(\delta)$. Despite its importance for the study of C\&P, Wäscher, Haußner, and Schumann (2007, p.1112) identified a number of weaknesses in this notation, stating that it 'does not necessarily result in homogeneous problem categories' and noted that it contains a 'partially inconsistent' categorisation. Wäscher, Haußner, and Schumann (2007, p.1111) further note that its 'coding scheme was not self-explanatory from the view point of an international (English-speaking) community or researchers'.

Wäscher, Haußner, and Schumann (2007) aimed to address the identified issues by introducing a more consistent terminology and refined categorisation criteria, including the introduction of a basic, intermediate and refined categorisation for problems. Basic C\&P problems contain the definition of the kind of assignment or objective and the assortment of small items, which can

\footnotetext{
${ }^{1}$ https://paginas.fe.up.pt/ esicup/datasets (last accessed on 1 July 2017)

${ }^{2}$ http://people.brunel.ac.uk/ mastjjb/jeb/info.html (last accessed on 1 July 2017)
} 
be identical, weakly or strongly heterogeneous. Intermediate problems provide information on containers, and refined problems also include information about the dimensionality, the shape of the insertable items and optional constraints.

The classification and representation of packing problems depends, among other aspects, on the assortment of items or parts and their features. Dyckhoff's (1990) typology, for example, distinguishes between parts in terms of their shape, which can be either regular (forms that can be described by few parameters, e.g. rectangles or spheres) or irregular. In contrast, Wäscher, Haußner, and Schumann (2007) discriminated by the number of types of items, these being weakly or strongly heterogeneous. This nomenclature, however, depends on the interpretation of linguistic terms such as 'few' or 'many' to describe variation.

A more detailed classification for two and three-dimensional rectangular geometries was provided by Egeblad and Pisinger (2009), who categorised parts into five classes: flat items, long items, cubes, uniform items (in which the greatest dimension is no more than twice the smallest) and diverse items (in which the greatest dimension can reach 50 times the smallest). Of course, these categorisation criteria ignore important geometric features of relevance to irregular spatial packing problems, such as convexity, relative complexity and multiplicity.

\subsection{An alternative taxonomy for packing problems}

To address the limitations of the existing taxonomies, this section presents a taxonomy for 3DIP problems that is comprised of a terminology and categorisation criteria for problems and instances, which can be used to realistically describe 3DIP problems found in AM. A taxonomy is a useful resource for researchers and practitioners since an ambiguous terminology or imprecise classification criteria might lead to miscommunication. The terms nesting and packing, for example, have sometimes been used interchangeably, although nesting is normally associated with a pattern in which some larger objects envelope one or more smaller objects. To eliminate this ambiguity, the following introduces a consistent terminology, which is in accordance with the usual nomenclature employed in the AM community:

- Model: A comprehensive definition of an object such that the construction of multiple objects of that type is possible by referring only to the definition. In the AM context, a model is usually provided as a three-dimensional mesh in the STL, or similar, format (Gardan 2016). From a given digital model in this format, it is possible to calculate its volume, surface area and the number of triangles, or faces.

- Part: A part or item is a single physical instantiation of a model. Extant taxonomies (Dyckhoff 1990; Wäscher, Haußner, and Schumann 2007) used the term 'small item' instead.

- Multiplicity: Quantities of parts to be produced per model.

- Demand profile: Additional information describing a client's requirements regarding parts, such as the due date or profit values.

- Container: The geometry and dimensions of the volume available to accommodate the requested parts. In AM, this corresponds to the specifications of the available space within the AM system and can be associated with build speed and cost parameters.

- Build: A group of parts produced simultaneously by an AM system at a particular time in a single operation. The assignment of a part to a particular build is constrained by volume capacity and geometrical non-overlapping conditions.

- Time horizon: Estimated length of time necessary to complete the production, which can be continuous or discretised into equal time intervals.

- Problem instance: Refers to a single input problem and encloses the information of one or more containers or parts, multiplicity, demand profile and, optionally, a planning horizon that requires the specification of the due dates for the requested parts.

- Dataset: A set of problem instances for assessing and comparing solution methods. 
Table 1. Summary of the four-tuple $D|C| B \mid$ Ato describe 3DIP problems.

\begin{tabular}{ccc}
\hline Element & Description & Values \\
\hline$D$ & Dimensionality of the problem & $1,2,2+1$ or 3 \\
\hline$C$ & Criteria for optimisation & $O u, S i, C p$ or $T p$ \\
\hline$B$ & Build volume types & $O f, O o, I$ or $H$ \\
\hline$A$ & Attributes/features of the assortment of parts & $l l, h l, l h, h h$ \\
\hline
\end{tabular}

\subsection{Categorisation of three-dimensional irregular packing problems}

The proposed categorisation criteria and notation refine the most recent taxonomy (Wäscher, Haußner, and Schumann 2007) and capture 3DIP problems using four-tuples $D|C| B \mid A$. Although there are similarities to the $\alpha / \beta / \gamma / \delta$ format proposed by Dyckhoff (1990), this formulation is intended to address the aforementioned issues with the previous taxonomies in addition to including further information regarding AM-related objectives and features of the problem. Table 1 summarises the encoding scheme that is described in this section.

The first element in the four-tuple $(D)$ denotes the dimensionality of the problem, which is usually three dimensional (' 3 ') due to the nature of packing problems in AM. Some solution methods (Canellidis et al. 2006; Canellidis, Giannatsis, and Dedoussis 2009), however, reduce the input problem to a two-dimensional instance where the XY-projection of items must be arranged on the build platform or substrate. Dyckhoff (1990) used the notation $2+1$ to refer to the dimensionality of this kind of problem; in AM operations this is applicable to technology variants that require sacrificial support structures, such as material jetting, resin vat processes or material extrusion.

The optimisation criterion $(C)$ identifies one of the following objectives: Output maximisation $(\mathrm{Ou})$, Single input minimisation $(S i)$, Time-minimisation parallel production $(T p)$ or Costminimisation parallel production $(C p)$. Output maximisation $(O u)$ describes a problem where the demand for parts is higher than the container capacity and has as objective to pack the subset of items that maximises the total volume of packed parts. This optimisation criterion occurs in a 3DIP variant involving a single build volume and unspecified time horizon.

Single input minimisation $(\mathrm{Si})$ and describes problems that address a single hypothetical container with one or more variable-length dimensions and the objective of finding a configuration for items that minimises the container's variable dimensions. In practical cases, this problem occurs when it is known that the entire set of parts can be accommodated within the container (Wäscher, Haußner, and Schumann 2007), which is a requirement that is not always true.

The optimisation criteria of cost-minimisation parallel production $(C p)$ and time-minimisation parallel production $(T p)$ comprise more complex scenarios where multiple builds are necessary to manufacture the requirement for parts, as defined by demand. For problems where the containers have the same estimated building cost, $C p$ is equivalent to the minimisation of the number of builds - the classic Bin Packing Problem (Wäscher, Haußner, and Schumann 2007). For the timeminimisation parallel production criterion, the due time and completion time of parts carries greater importance (Tavakkoli-Moghaddam et al. 2005). This involves the minimisation of one or more of the following variables: the makespan (time necessary to manufacture the entire demand), the total tardiness, or the earliness. Tardiness and earliness are computed in terms of the differences between the completion times and due times of required parts, and the solution of this type of problem requires the combination of scheduling and three-dimensional irregular packing techniques. There has been only sparse study related to this class of problems to the present day (Lawler et al. 1993; Iori and Martello 2010).

The third element of the $D|C| B \mid A$ notation $(B)$ is a descriptor of the attributes of the container or containers available in the problem. It is proposed that this specification corresponds to one of the following: one container with fixed dimensions $(O f)$, one container with open Z-height $(O o)$, or 
Table 2. Features of the assortment of parts $(A)$ based on relative standard deviation (RSD) of demands and mean connectivity value (MCV).

\begin{tabular}{lccl}
\hline$A$ & $\begin{array}{c}\text { Demand } \\
\text { variation }\end{array}$ & $\begin{array}{c}\text { Mean } \\
\text { complexity }\end{array}$ & Common solution \\
\hline $\begin{array}{l}\text { Low demand variation }+ \\
\text { low mean complexity }(l l)\end{array}$ & $\begin{array}{c}\text { RSD } \in \\
{[0,0.5]}\end{array}$ & $\begin{array}{c}\text { MCV } \in \\
{[0,0.5]}\end{array}$ & $\begin{array}{l}\text { Iterative application of placement heuristics such as Deepest } \\
\text { Bottom-Left (Baumers et al. 2013). }\end{array}$ \\
\hline $\begin{array}{l}\text { Low demand variation }+ \\
\text { high mean complexity }(l h)\end{array}$ & $\begin{array}{c}\text { RSD } \in \\
{[0,0.5]}\end{array}$ & $\begin{array}{c}\text { MCV } \in \\
(0.5,1]\end{array}$ & $\begin{array}{l}\text { Parts can be simplified into regular (often rectangular) geome- } \\
\text { tries and then solved by exact methods (Stoyan et al. 2005). }\end{array}$ \\
\hline $\begin{array}{l}\text { High demand variation }+ \\
\text { low mean complexity }(h l)\end{array}$ & $\begin{array}{c}\text { RSD } \\
>0.5\end{array}$ & $\begin{array}{c}\text { MCV } \in \\
{[0,0.5]}\end{array}$ & $\begin{array}{l}\text { This type of problem has not been systematically addressed } \\
\text { in the literature. }\end{array}$ \\
\hline $\begin{array}{l}\text { High demand variation }+ \\
\text { high mean complexity }(h h)\end{array}$ & RSD & $\begin{array}{c}\text { MCV } \in \\
(0.5,1]\end{array}$ & $\begin{array}{l}\text { Reduction of the parts to their minimum bounding boxes and } \\
\text { application of wall-building heuristics (Canellidis et al. 2006). }\end{array}$ \\
\hline
\end{tabular}

variable-length dimension, multiple identical containers $(I)$ or multiple heterogeneous containers $(H)$. It is additionally assumed that the standard shape of the large object is rectangular or cuboid, for two and three-dimensions respectively, as usual in build volume specifications.

The last element $(A)$ is optional when specifying 3DIP problems, since it characterises the instance rather than the problem class, and adds information that might support the choice of a suitable solution method. It is proposed that Avaries according to the demand variation and average part complexity, assuming one of the following four permutations: low demand variation + low mean complexity $(l l)$, high demand variation + low mean complexity $(h l)$, low demand variation + high mean complexity $(l h)$, high demand variation + high mean complexity $(h h)$, or simply $A$ to describe the presence of multiple instances with different characteristics or unknown feature values.

This paper also proposes to address the issue of subjective evaluations of 'low' and 'high' by determining the characteristics of the problem instance $(A)$ through the use of relative standard deviation (RSD) of the multiplicities, which is a measurable feature formed by the ratio of the standard deviation and mean of demand quantities. Low demand variations are here defined as those which have values equal to or less than 0.5 ; high variations have an RSD greater than 0.5.

Further, two complexity metrics are used in this analysis, which are the Spies Ratio and the Mean Connectivity Value. As complexity is an inherently multi-faceted notion, many other metrics based on other interpretations of complexity are imaginable (Gell-Mann 2002). The Spies Ratio (SR) is a traditional complexity measure used in manufacturing, which is obtained by dividing the volume of the geometry part by the volume of the primitive that contains it (Spies 1957). Araújo et al. (2016) proposed a practical computation-based method for the calculation of the Spies Ratio by dividing the volume of the part by the volume of its minimum bounding box, as optimised through a local search algorithm. This complexity metric ranges between approximately zero for complex parts and one for entirely convex primitive geometries.

A complexity metric that has been applied in empirical work to AM (Araújo et al. 2016; Baumers et al. 2016) is the mean connectivity value (MCV). Originally proposed to capture complexity in architecture (Psarra and Grajewski 2001), this metric is able to support AM processes by analysing geometries according to the frequency and regularity with which concavities and entry features occur. In the specification of part assignment, a lower complexity measurement exhibits an MCV less than or equal to 0.5 while a high complexity measurement has MCV in the interval $(0.5,1]$. Table 2 presents the values for $A$ according to demand variation and mean complexity, and conventional approaches that can be used to solve each category. 


\section{Datasets for 3D irregular packing problems}

This section reviews existing 3DIP techniques, datasets and the corresponding features. A new dataset is also introduced in answer to the identified need for more realistic benchmarking in AM, which consists of a set of digital resources that enable researchers to execute benchmark tests on different 3DIP approaches. This forms a valuable tool to assess, compare and identify the relative strengths and weaknesses of packing algorithms.

\subsection{Approaches and datasets for 3DIP problems in $A M$}

In comparison to three-dimensional regular packing problems, there are few solution techniques and datasets for 3DIP in the scientific literature that are fully aligned with the real-world AM problems (Araújo et al. 2015). This section reviews recent studies of 3DIP problems in AM between 2000 and 2016 and the datasets utilised to assess their efficiency, as summarised in Table 3.

The addressed 3DIP problems have been categorised in Table 3 according to the introduced notation. To illustrate the need for a new taxonomy, it is interesting to note that the problem considered by Egeblad, Nielsen, and Brazil (2009) could be represented using Dyckhoff's taxonomy (1990) as any of the following formations depending on the reader's judgement: $3 / \mathrm{V} / \mathrm{O} / \mathrm{M}$, $3 / V / O / R, 3 / V / D / M, 3 / V / D / R$, where $V$ means that all items must be arranged into a selection of containers; $O$, presence of only one container; $D$, different containers; $M$, many items of many models; $R$, many items of few models. Not only can this result in potential confusion, but it also provides less useful information about the structural details to the person tasked with doing the packing.

As stated in the introduction, these packing problems are NP-Hard and not easily modelled (Garey and Johnson 1979). Hence, most of the existing solutions are based on heuristics or metaheuristics. For example, some methods assign random positions to the parts and then successively apply local search algorithms that perform perturbations (small variations of position or orientation) on a subset of parts to improve the overall volume utilisation (Lutters, ten Dam, and Faneker 2012; Verkhoturov et al. 2016).

Most 3DIP solutions, however, utilise variations of the Deepest Bottom-Left (DBL) technique. DBL is a procedure that inserts a pre-determined sequence of parts, one at a time, into the container and manipulates each part to find the deepest and most bottom-left position through translation and rotation. This method is usually employed in conjunction with a strategy for ordering the parts, based on part size or priority (Baumers et al. 2013), for example. An alternative methodology to search for patterns in the sequence of parts is the utilisation of Genetic Algorithms (GA), which have been applied to AM in the past (Ikonen et al. 1997; Canellidis et al. 2006; Gogate and Pande 2008; Canellidis, Giannatsis, and Dedoussis 2010; Wu et al. 2014).

An additional issue in the development of solutions for 3DIP in the context of AM is the scarcity of available datasets. As noted by Egeblad, Nielsen, and Brazil (2009, p.283) the literature on the subject of three-dimensional packing contains only a few useful problem instances with regard to a comparison of results. To aid reader orientation, Table 4 introduces a shorthand notation for each dataset identified in the literature, combining the first letter of the first author's surname and the year of publication. For example, I1997 denotes the dataset provided by Ikonen et al. (1997). In the following discussions, particular instances drawn from the datasets are identified by adding a suffix to the shorthand notation, denoting the instance number (e.g. I1997:1).

Figure 1 illustrates the characteristics of the models in the datasets described in Table 4 in terms of the number of faces of the contained geometries (Figure 1a) and the part complexity (as measured by MCV, Figure 1b). The distribution reveals that only a few models are formed by more than 30,000 faces. For example, the datasets W2014 and B2013 contain, respectively, only 1 and 2 models exceeding 30,000 faces. Regarding the part complexity measured by MCV, Figure 
Table 3. 3DIP studies published between 2000 and 2017.

\begin{tabular}{|c|c|c|c|c|}
\hline Reference & $\mathrm{AM}^{\mathrm{a}}$ & Problem $^{\mathrm{b}}$ & Dataset $^{\mathrm{c}}$ & Solution approach \\
\hline Hur et al. (2001) & LS & $3|S i| O o \mid A$ & own ${ }^{d}$ & DBL; GA \\
\hline Ravindran (2003) & $\mathrm{n} / \mathrm{a}$ & $3|C p| I \mid A$ & own ${ }^{d}$ & Octree discretisation; GA \\
\hline Stoyan et al. (2004) & $\mathrm{n} / \mathrm{a}$ & $3|S i| O o \mid A$ & own $^{\mathrm{d}}$ & Mathematical modelling \\
\hline Stoyan et al. (2005) & $\mathrm{n} / \mathrm{a}$ & $3|S i| O o \mid A$ & own & Mathematical modelling \\
\hline Canellidis et al. (2006) & SLA & $2+1|O u| O f \mid l l$ & own & DBL; GA \\
\hline Egeblad, Nielsen, and Odgaard (2007) & $\mathrm{n} / \mathrm{a}$ & $3|S i| O o \mid l h$ & $\mathrm{I} 1997$ & Greedy Local Search \\
\hline Gogate and Pande (2008) & LS & $3|S i| O o \mid l h$ & own & DBL; GA \\
\hline Egeblad (2009) & $\mathrm{n} / \mathrm{a}$ & $3|S i| O o \mid A$ & $\mathrm{~S} 2005 ;$ own $^{\mathrm{d}}$ & Local Search; Guided Local Search \\
\hline Egeblad, Nielsen, and Brazil (2009) & $\mathrm{LS}$ & $3|S i| O o \mid A$ & $\mathrm{~S} 2005 ;$ own $^{\mathrm{d}}$ & Local Search; Guided Local Search \\
\hline Lee et al. (2009) & $\mathrm{n} / \mathrm{a}$ & $3|S i| O o \mid A$ & own $^{\mathrm{d}}$ & Local Search \\
\hline Canellidis, Giannatsis, and Dedoussis (2010) & SLA & $2+1|O u| O f \mid l l$ & own & DBL; GA \\
\hline Chernov, Stoyan, and Romanova (2010) & $\mathrm{n} / \mathrm{a}$ & $3|S i| O o \mid A$ & own $^{\mathrm{d}}$ & Mathematical modelling \\
\hline Stafford and Jackson (2010) & $\mathrm{n} / \mathrm{a}$ & $3|O u| O f \mid A$ & own ${ }^{\mathrm{d}}$ & Local Search \\
\hline Lutters, ten Dam, and Faneker (2012) & $\mathrm{LS}$ & $3|S i| O o \mid A$ & own $^{d}$ & Local Search \\
\hline Baumers et al. (2013) & $\mathrm{LS}$ & $3|O u| O f \mid l l$ & own & DBL \\
\hline Canellidis, Giannatsis, and Dedoussis (2013) & SLA & $2+1|O u| O f \mid l l$ & own $^{\mathrm{d}}$ & DBL; GA \\
\hline Wu et al. (2014) & $\mathrm{LS}$ & $3|S i| O o \mid l h$ & G2008; own & $\mathrm{DBL} ; \mathrm{GA}$ \\
\hline Liu et al. (2015) & $\mathrm{n} / \mathrm{a}$ & $3|S i| O o \mid A$ & S2004 & Local Search based \\
\hline Verkhoturov et al. (2016) & LS & $3|S i| O o \mid A$ & $\mathrm{~S} 2005$ & Discretisation; Greedy Local Search \\
\hline
\end{tabular}

a AM application: Laser Sintering (LS) or Stereolithography (SLA).

${ }^{\mathrm{b}}$ Problem in the proposed notation.

${ }^{\mathrm{c}}$ Dataset including shorthand.

dUnavailable dataset.

$1 \mathrm{~b}$ shows that none of the datasets cover the entire range of values for this feature. The datasets C2010 and B2013, for instance, do not contain any parts with MCV between 0.8 and 1.

In addition to the analysis of the models, it is equally advantageous to investigate the features of the instances. An overview of the features of the instances in the considered datasets, shown in Table 4, corroborates the necessity for a more heterogeneous 3DIP problem dataset. The first observation drawn from Table 4 is the predominance of $l l$ instances with regards to the assortment of parts $(A)$. The second observation is that few (only 2 out of 13) instances are comprised of more than 25 parts, which is one of the main characteristics that affect algorithm performance.

Table 4 shows that none of the instances exhibits a variation of complexity (RSD of MCV) in the upper half of its range, while most instances have a mean MCV around 0.4. It can be observed that only five instances have an RSD of demand greater than zero. At the same time, most instances exhibit a high volume variation, with an RSD of volume greater than 0.5 . This low complexity and low variability is not usually observed in real packing problems. Indeed, the ability to handle complex models is one of the main benefits of AM. The overall conclusion is that the combinations of values of features do not cover the entire range of permutations, leaving some categories unrepresented. 


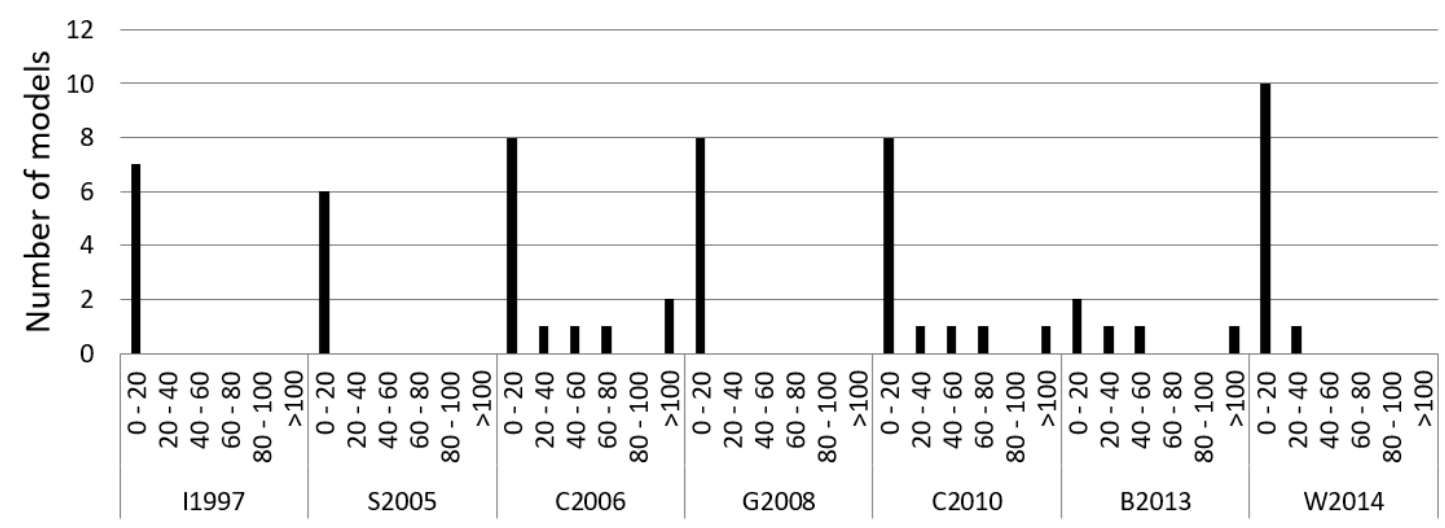

Range of number of faces

(a) Number of models in relation to the range of the number of faces, in thousands.

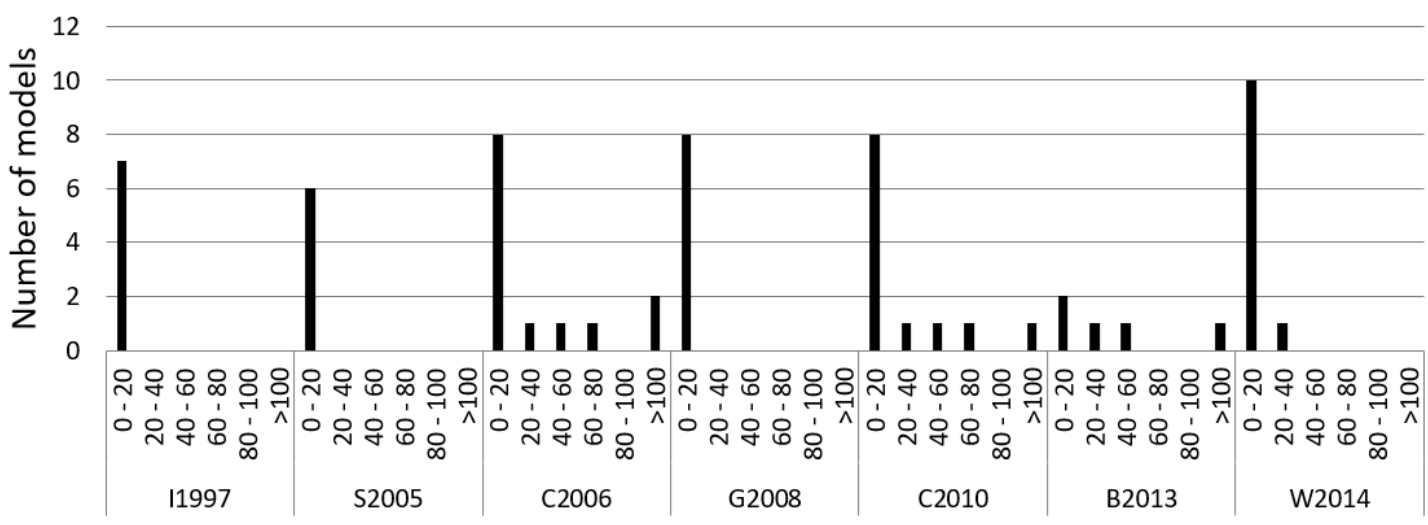

Range of number of faces

(b) Number of models in relation to the range of the MCV.

Figure 1. Histograms of two features for models in the previous datasets.

\subsection{A new dataset for 3DIP problems in AM}

The new dataset ${ }^{3}$, which we will refer to as A2018, was created with the purpose of providing an extensive number of packing problems that better reflects realistic scenarios in AM. In this dataset, each instance is provided in two formats: extensible markup language (XML) files and serialised Java objects. Furthermore, each instance contains the description of the container(s), set of models and demands, parts and respective due dates. Together, the combination of the representation of the input problems and the achieved configuration of parts as XML files and Java source code provides a valuable tool for use in the early stages of development of problem solutions, containing a much larger number of real-world geometries than were available in previous datasets.

The parts in the proposed dataset are now analysed with respect to the features presented, as in Section 2.2. Figure 2 shows histograms of the number of faces and the MCV for the 435 models in A2018.

Figure 2a shows that dataset A2018 contains the highest number of models with more than 10,000 faces. Moreover, all ranges of values for the number of faces and MCV are represented, as shown in Figure 2b. The existing datasets have a limited number of instances, which each exhibit a

\footnotetext{
${ }^{3}$ http://www.cs.nott.ac.uk/ psxlja/dataset
} 
Table 4. Features of the instances in the extant 3DIP datasets.

\begin{tabular}{lccccccccc}
\hline Instance & $A$ & $\begin{array}{c}\text { \# of } \\
\text { parts }\end{array}$ & $\begin{array}{c}\text { \# of } \\
\text { models }\end{array}$ & $\begin{array}{c}\text { RSD of } \\
\text { demand }\end{array}$ & $\begin{array}{c}\text { Mean } \\
\text { MCV }\end{array}$ & $\begin{array}{c}\text { RSD of } \\
\text { MCV }\end{array}$ & $\begin{array}{c}\text { Mean } \\
\text { SR }\end{array}$ & $\begin{array}{c}\text { RSD of } \\
\text { SR }\end{array}$ & $\begin{array}{c}\text { RSD of } \\
\text { volume }\end{array}$ \\
\hline I1997:1 & $l h$ & 7 & 7 & 0.00 & 0.73 & 0.37 & 0.45 & 0.81 & 1.05 \\
\hline S2005:1 & $l h$ & 7 & 7 & 0.00 & 1.00 & 0.00 & 0.48 & 0.56 & 0.23 \\
\hline S2005:2 & $h h$ & 12 & 7 & 0.55 & 1.00 & 0.00 & 0.42 & 0.56 & 0.18 \\
\hline S2005:3 & $l h$ & 25 & 7 & 0.39 & 1.00 & 0.00 & 0.52 & 0.50 & 0.22 \\
\hline C2006:1 & $l l$ & 14 & 14 & 0.00 & 0.35 & 0.37 & 0.12 & 0.75 & 0.93 \\
\hline G2008:1 & $l h$ & 9 & 9 & 0.00 & 0.96 & 0.11 & 0.54 & 0.55 & 0.67 \\
\hline C2010:1 & $l l$ & 13 & 13 & 0.00 & 0.29 & 0.42 & 0.14 & 0.65 & 1.05 \\
\hline B2013:1 & $h l$ & $85^{\text {a }}$ & 5 & 1.71 & 0.39 & 0.11 & 0.32 & 0.41 & 1.32 \\
\hline B2013:2 & $l l$ & 25 & 5 & 0.00 & 0.39 & 0.11 & 0.32 & 0.41 & 1.32 \\
\hline B2013:3 & $l l$ & 15 & 5 & 0.00 & 0.39 & 0.12 & 0.32 & 0.42 & 1.34 \\
\hline B2013:4 & $l l$ & 5 & 5 & 0.00 & 0.39 & 0.13 & 0.32 & 0.44 & 1.44 \\
\hline B2013:5 & $l l$ & $3^{\text {a }}$ & 1 & 0.00 & 0.44 & 0.00 & 0.19 & 0.00 & 0.00 \\
\hline B2013:6 & $l l$ & $23^{\text {a }}$ & 2 & 0.18 & 0.34 & 0.03 & 0.38 & 0.55 & 1.14 \\
\hline B2013:7 & $h l$ & $145^{\text {a }}$ & 2 & 0.53 & 0.42 & 0.05 & 0.34 & 0.37 & 1.24 \\
\hline B2013:8 & $l l$ & 1 & 1 & 0.00 & 0.44 & 0.00 & 0.19 & 0.00 & 0.00 \\
\hline W2014:1 & $l h$ & 12 & 12 & 0.00 & 0.73 & 0.27 & 0.43 & 0.66 & 1.44 \\
\hline
\end{tabular}

${ }^{a}$ Number of successfully packed parts by Baumers et al. (2013), which presents infinity demands requirements.

low number of parts (typically $<20$ ). The new dataset, on the other hand, increases the challenge of solving the 3DIP problem since the contained models possess well-dispersed characteristics as illustrated in Figure 2c and Figure 2d. Moreover, the 2,343 instances in A2018 cover the entire range of values for mean and relative standard deviation of MCV and SR as presented in Figures $2 \mathrm{e}$ and $2 \mathrm{f}$.

\subsection{Applying packing heuristics}

This section illustrates the usefulness of the new dataset (A2018) in the assessment of various 3DIP techniques by performing a computational experiment utilising a subset of 13 selected instances, as detailed in Table 5. These are solved by employing two well-known packing heuristics. The first method is the Deepest Bottom Left with Fill (DBLF) (Karabulut and İnceoğlu 2004). DBLF was combined with the First Fit Decreasing (FFD) heuristic, which sorts the parts in decreasing order by their volumes and then allocates one part at a time to the first possible position. The algorithm employed in the experiment used the native orientation of parts, no additional rotations were applied to the geometries. The second technique investigates a wall-building based method (WBA) (Pisinger 2002) that arranges the bounding boxes of geometries, which can be rotated orthogonally, into horizontal and vertical layers successively until the container is filled (Araújo and Pinheiro 2010).

In this experiment, each instance was solved by two solution approaches, which aim to address different AM problems. The first problem is associated with the AM technology variant Stereolithography (Jacobs 1992) and is denoted as $2+1|O u| O f \mid A$ in the classification presented in this paper, amounting to a two-dimensional cutting problem of the vertical projection of parts. The second problem reflects the operation of the AM technology variant Laser Sintering (Gibson, Rosen, and Stucker 2014) and is represented by the notation $3|O u| O f \mid A$, allowing the unconstrained positioning of parts in three-dimensional space. As an example, the first instance (A2018:0004) was 


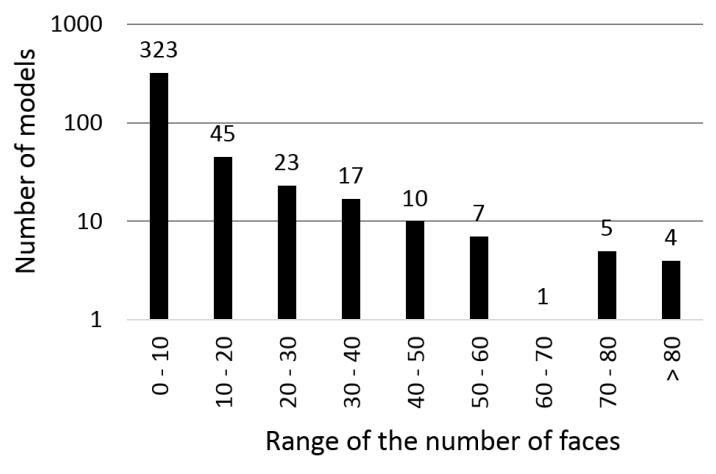

(a) Number of models in relation to number of faces.

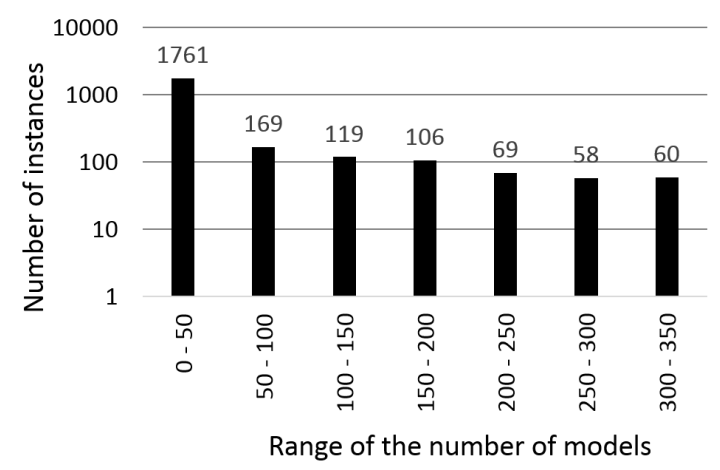

(c) Number of instances in relation to number of models.

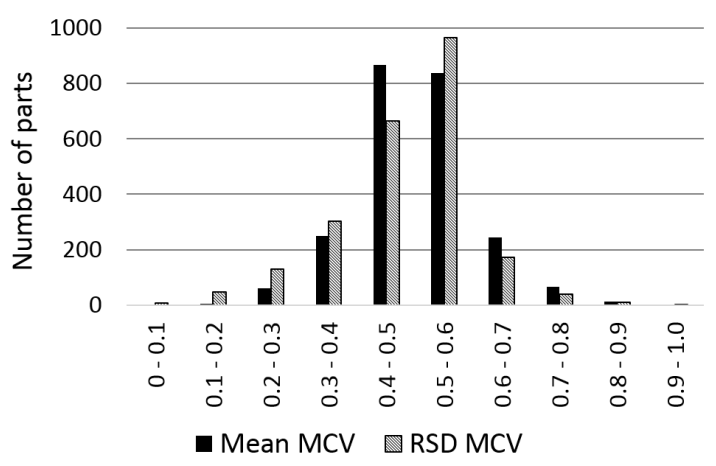

(e) Number of parts per mean and RSD of MCV.

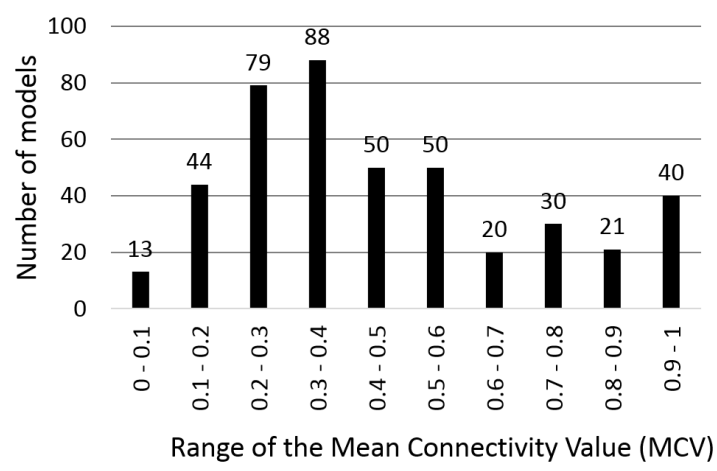

(b) Number of models in relation to MCV.

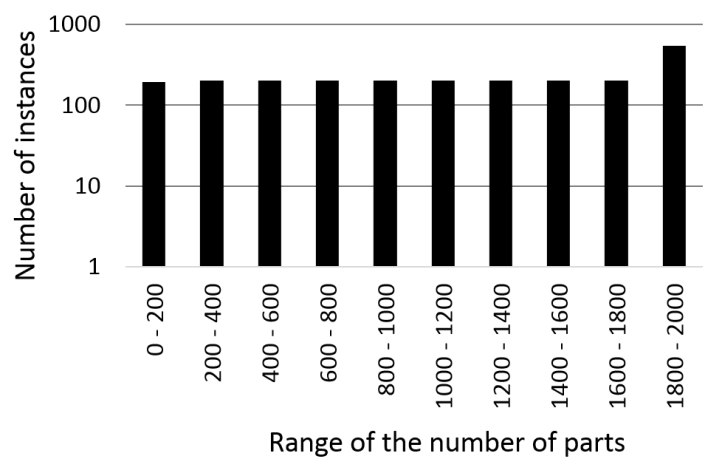

(d) Number of instances in relation to number of parts.

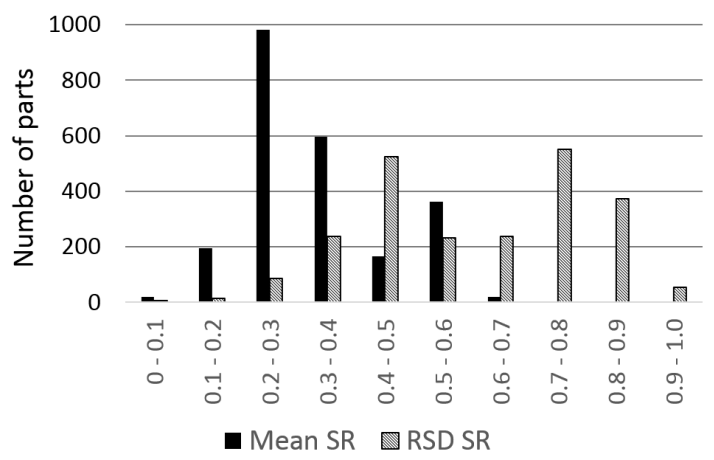

(f) Number of parts per mean and RSD of SR.

Figure 2. Histograms of features for models and instances in the new dataset.

solved by a DBLF algorithm according to the constraints and objectives of $2+1|O u| O f \mid l l$ problems. Table 6 presents the build volume utilisation (BVU) and running time (in seconds) obtained by employing DBLF and the WBA for the selected instances and problems. Figure 3 illustrates the graphical visualisation output obtained from the different packing algorithms for the same instances and problems.

At this point, it is appropriate to investigate possible correlations between the features of instances, key performance indicators of the algorithms and characteristics of the packing problem addressed. Table 7 shows the correlations with the performance indicators: build volume utilisation and running time of DBLF and WBA.

The use of correlation coefficients allows the reader to extract useful insights regarding the 
Table 5. A sample of 13 instances from A2018 and their respective features.

\begin{tabular}{ccccccccccc}
\hline Instance & $A$ & $\begin{array}{c}\# \text { of } \\
\text { parts }\end{array}$ & $\begin{array}{c}\text { \# of } \\
\text { models }\end{array}$ & $\begin{array}{c}\text { RSD of } \\
\text { demands }\end{array}$ & $\begin{array}{c}\text { Mean } \\
\text { MCV }\end{array}$ & $\begin{array}{c}\text { RSD of } \\
\text { MCV }\end{array}$ & $\begin{array}{c}\text { Mean } \\
\text { SR }\end{array}$ & $\begin{array}{c}\text { RSD } \\
\text { of SR }\end{array}$ & $\begin{array}{c}\text { RSD of } \\
\text { volumes }\end{array}$ & $\begin{array}{c}\text { Mean \# } \\
\text { of faces }\end{array}$ \\
\hline A2018:0004 & $l l$ & 9 & 8 & 0.294 & 0.421 & 0.344 & 0.248 & 0.867 & 1.040 & 2794.9 \\
\hline A2018:0007 & $l l$ & 12 & 9 & 0.354 & 0.471 & 0.517 & 0.289 & 0.815 & 1.025 & 2892.3 \\
\hline A2018:0008 & $l l$ & 13 & 5 & 0.308 & 0.487 & 0.528 & 0.312 & 0.774 & 0.902 & 8117.4 \\
\hline A2018:0009 & $l l$ & 14 & 4 & 0.319 & 0.316 & 0.286 & 0.253 & 0.895 & 0.872 & 4724.3 \\
\hline A2018:0010 & $l h$ & 15 & 14 & 0.240 & 0.527 & 0.339 & 0.372 & 0.537 & 0.521 & 1004.7 \\
\hline A2018:0015 & $h h$ & 20 & 8 & 0.600 & 0.597 & 0.561 & 0.486 & 0.380 & 0.898 & 4231.6 \\
\hline A2018:0020 & $l h$ & 25 & 8 & 0.492 & 0.590 & 0.344 & 0.337 & 0.656 & 0.792 & 9902.5 \\
\hline A2018:0021 & $l h$ & 26 & 14 & 0.493 & 0.511 & 0.436 & 0.393 & 0.633 & 0.922 & 2681.2 \\
\hline A2018:0023 & $l h$ & 28 & 8 & 0.319 & 0.519 & 0.531 & 0.239 & 0.972 & 1.291 & 6823.2 \\
\hline A2018:0026 & $h l$ & 31 & 13 & 0.582 & 0.438 & 0.539 & 0.221 & 0.687 & 0.853 & 6791.3 \\
\hline A2018:0028 & $h h$ & 33 & 8 & 0.599 & 0.76 & 0.298 & 0.356 & 0.820 & 1.759 & 7510.2 \\
\hline A2018:0046 & $h h$ & 51 & 25 & 0.643 & 0.523 & 0.522 & 0.376 & 0.626 & 0.957 & 6267.2 \\
\hline A2018:0051 & $h l$ & 56 & 38 & 0.533 & 0.421 & 0.583 & 0.299 & 0.650 & 0.997 & 4794.8 \\
\hline
\end{tabular}

Table 6.

Build volume utilisation (BVU) and running time from two packing approaches - the Deepest Bottom Left with Fill (DBLF) (Karabulut and İnceoğlu 2004) and a wall-building based method (WBA) (Araújo and Pinheiro 2010)

\begin{tabular}{ccccccccc}
\hline \multicolumn{4}{c}{$2+1|O u| O f \mid A$} & \multicolumn{3}{c}{$3|O u| O f \mid A$} \\
\hline Instance & BVU & Time $^{\mathrm{a}}$ & BVU & Time $^{\mathrm{a}}$ & BVU & Time $^{\mathrm{a}}$ & BVU & Time $^{\mathrm{a}}$ \\
\hline A2018:0004 & 0.159 & 154.4 & 0.166 & 0.5 & 0.181 & 707.8 & 0.166 & 3.9 \\
\hline A2018:0007 & 0.073 & 19.6 & 0.150 & 1.4 & 0.108 & 190.9 & 0.150 & 14.6 \\
\hline A2018:0008 & 0.062 & 1226.2 & 0.086 & 0.4 & 0.062 & 19878.6 & 0.079 & 1.9 \\
\hline A2018:0009 & 0.125 & 994 & 0.185 & 0.5 & 0.125 & 2317.4 & 0.148 & 0.2 \\
\hline A2018:0010 & 0.127 & 155 & 0.199 & 3.2 & 0.173 & 1786.2 & 0.194 & 2.5 \\
\hline A2018:0015 & 0.123 & 826.8 & 0.237 & 2.0 & 0.228 & 2041 & 0.237 & 87.6 \\
\hline A2018:0020 & 0.065 & 19743.4 & 0.116 & 1.0 & 0.065 & 217498.8 & 0.116 & 1.4 \\
\hline A2018:0021 & 0.037 & 1004.7 & 0.515 & 3.7 & 0.037 & 3595.3 & 0.515 & 105.8 \\
\hline A2018:0023 & 0.132 & 11670.4 & 0.499 & 1.0 & 0.140 & 36818.7 & 0.502 & $1200^{\mathrm{b}}$ \\
\hline A2018:0026 & 0.199 & 2306.8 & 0.239 & 1.2 & 0.214 & 37915.7 & 0.270 & $1200^{\mathrm{b}}$ \\
\hline A2018:0028 & 0.379 & 15990.6 & 0.396 & 2.5 & 0.396 & 99380.9 & 0.396 & $1200^{\mathrm{b}}$ \\
\hline A2018:0046 & 0.426 & 18905.4 & 0.547 & 1.4 & 0.484 & 151196.7 & 0.619 & $1200^{\mathrm{b}}$ \\
\hline A2018:0051 & 0.347 & 5203 & 0.328 & 1.7 & 0.370 & 13290.4 & 0.360 & $1200^{\mathrm{b}}$ \\
\hline
\end{tabular}

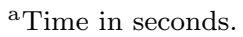

b Time limit for the WBA.

relationship between the variables involved in the problem. The calculated correlations show that, for example, a strong correlation between the running time of DBLF and the mean number of faces in a given problem, regardless of the problem being addressed (whether it is $2+1|O u| O f \mid A$ or $3|O u| O f \mid A)$. This simple observation reinforces the argument that the collision detection between faces of irregular parts contributes to the poor performance of DBLF. Another insight obtained from the experiment is the strong correlation between the BVU and the number of parts for different 


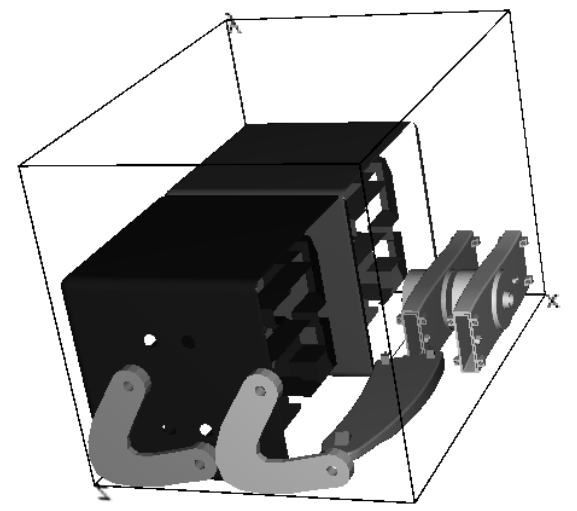

(a) Solution achieved by DBLF for A2018:0046 under $2+1|O u| O f \mid h h$

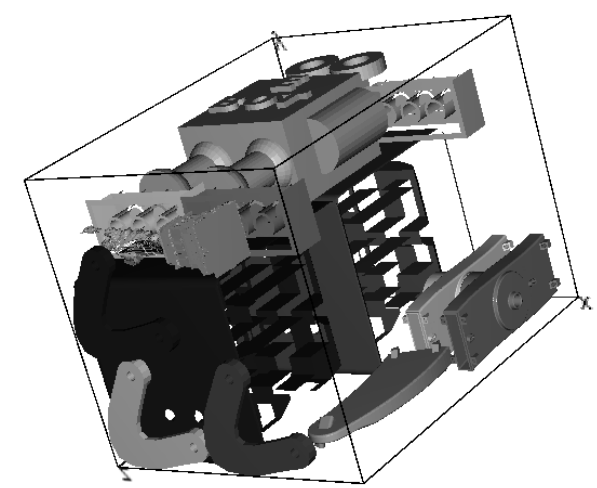

(c) Solution achieved by DBLF for A2018:0046 under $3|O u| O f \mid h h$

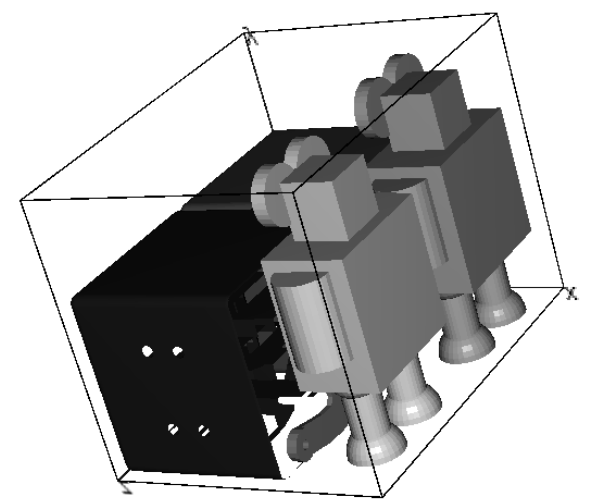

(b) Solution achieved by WBA for A2018:0046 under $2+1|O u| O f \mid h h$

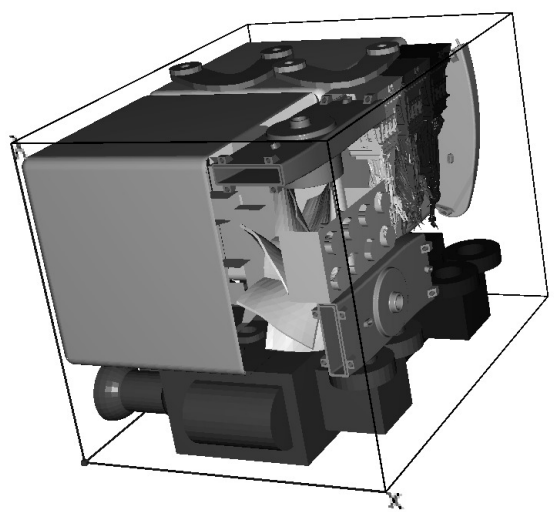

(d) Solution achieved by WBA for A2018:0046 under $3|O u| O f \mid h h$

Figure 3. Examples of solutions found by the DBLF and WBA in the experiment.

Table 7. Correlations between instance features, key performance indicators of packing algorithms and characteristics of the problems.

\begin{tabular}{ccccccccc}
\hline \multicolumn{1}{c}{ DBLF } & \multicolumn{5}{c}{ WBA } \\
\hline & $2+1|O u| O f \mid A$ & $3|O u| O f \mid A$ & \multicolumn{2}{c}{$2+1|O u| O f \mid A$} & $3|O u| O f \mid A$ \\
\hline BVU & Time & BVU & Time & BVU & Time & BVU & Time \\
\hline \# of parts & $\mathbf{0 . 7 8}$ & 0.56 & $\mathbf{0 . 7 4}$ & 0.4 & $\mathbf{0 . 6 4}$ & 0.18 & $\mathbf{0 . 7 0}$ & $\mathbf{0 . 8 1}$ \\
\hline \# of models & $\mathbf{0 . 6 2}$ & 0.16 & $\mathbf{0 . 6 1}$ & 0.07 & 0.42 & 0.25 & 0.49 & 0.53 \\
\hline RSD of demand & 0.59 & 0.48 & $\mathbf{0 . 6 2}$ & 0.47 & 0.45 & 0.24 & 0.51 & 0.55 \\
\hline Mean MCV & 0.24 & 0.55 & 0.28 & 0.48 & 0.26 & 0.45 & 0.25 & 0.21 \\
\hline RSD of MCV & 0.09 & -0.12 & 0.16 & -0.19 & 0.21 & -0.1 & 0.27 & 0.37 \\
\hline Mean SR & 0.04 & 0.1 & 0.18 & 0.13 & 0.19 & $\mathbf{0 . 6 1}$ & 0.19 & -0.22 \\
\hline RSD of SR & -0.03 & 0.08 & -0.18 & -0.06 & 0.01 & 0.52 & -0.04 & 0.15 \\
\hline RSD of volume & 0.46 & 0.41 & 0.39 & 0.14 & 0.42 & -0.02 & 0.38 & 0.53 \\
\hline Mean \# of faces & 0.17 & $\mathbf{0 . 6 9}$ & 0.09 & $\mathbf{0 . 7 2}$ & -0.02 & -0.47 & 0 & 0.36 \\
\hline
\end{tabular}

Strong correlations (greater than 0.5) are shown in bold. 
models, and the high variation of demand, regardless of the approach used for packing. The results obtained and the conclusions drawn here have been generated for illustration only, for the purpose of demonstrating potential utilisation of the dataset to observe the occurrence of patterns between the features of the instances, the characteristics of the problem and the solution method.

\section{Discussion}

As previously identified, the quality of the packing is important for managing costs. Hence, the selection of a packing approach affects the production performance frontier of AM (Schmenner and Swink 1998). In order to make a choice about the approach to use, it is important to understand which characteristics of the problem are important and which solution methods have been successfully applied to such problems in the past.

By proposing the extended taxonomy for 3DIP problems in AM, including a consistent terminology, a comprehensive notation and refined categorization criteria, this paper supports the development of theory regarding the efficient operation of highly flexible digital manufacturing technologies. As a central research product in both OM and OR, such formal theory consists of propositions that are not limited to particular contexts but are generally applicable (Glaser and Strauss 1967). By updating the existing taxonomy for 3DIP, we provide AM researchers with the vocabulary required to apply existing 3DIP theory to the emerging field of AM in order to determine features of relevance, as well as enabling OR and algorithm experts to identify potential applications for their research.

With an increased ability to flexibly manufacture complex and diverse geometries, the requirement to handle complexity in manufacturing control structures arises. This paper has aimed to eliminate some of the subjectivity introduced through the adoption of linguistic criteria, such as those proposed by Dyckhoff (1990) and Wäscher, Haußner, and Schumann (2007), regarding the categorisation of parts. Instead, this approach is based on the evaluation of measurable features residing in digital geometric models and the information systems supporting manufacturing. This is timely since digital manufacturing processes, such as AM, are based on virtual representations of products and processes, thereby cutting across the engineering and operations functions (Slansky 2008). Alongside the popularisation of the notion of 'Industrie 4.0' (Kagermann, Lukas, and Wahlster 2011), the reality of cheap and ubiquitous information processing, and with it networked production facilities drawing on real-time information, means that reactive and flexible processes are becoming central in manufacturing (Schwab 2017).

An example of such an application is the utilisation of hyper-heuristics for the solution of threedimensional regular packing problems (Allen et al. 2009). A well-known issue in heuristic optimisation is that different techniques often deliver dissimilar performances when applied to different instances. This phenomenon is observed in our experiments: for the $3|O u| O f \mid A$ problem, DBLF performs better than WBA on two instances, A2018:4 and A2018:51; the situation is, however, reversed in the remaining instances.

There is a growing number of studies on the use of data science techniques to discover a mapping from instance features to the best performing algorithm in problem solving (Ross 2005; Kotthoff 2014). By using the features of unseen instances and the discovered mapping, the best performing heuristic can be selected automatically to solve the given problem. Another strand of ongoing research which may have major benefits for this type of problem is on hyper-heuristics to mix and control multiple heuristics, with the goal of exploiting their relative strengths (Burke et al. 2013). An additional methodology that can be applied to 3DIP in the digital manufacturing context is machine learning, which studies algorithms designed to learn from data in order to make predictions. Machine learning has been adopted in flexible manufacturing technologies for a variety of purposes, ranging from specifying predictive maintenance scheduling, the generation of demand forecasts, to manufacturing process monitoring and optimisation (Joseph et al. 2014). 


\section{Conclusion}

The 3DIP problem captured in this paper sits at the heart of AM as a flexible manufacturing system capable of delivering a variety of complex products in a responsive and flexible way. The taxonomy proposed in this paper will aid the development of new solutions to this problem. The categorisation criteria and notation proposed forms a timely update of the traditional format developed by Dyckhoff (1990) and Wäscher, Haußner, and Schumann (2007), allowing researchers and practitioners to grasp the 3DIP problem as encountered in the AM context.

In future work, we expect that this will aid the identification of strategies for the assessment and comparison of solutions, subject to the objective criteria introduced in this paper. By also providing the benchmark required for such comparisons, we address specific practical needs: enabling researchers to test their solution approaches on more realistic problems, thus providing decision makers and AM experts with a better understanding of which solution approaches will be of more use for their specific problem characteristics. Ongoing activities that are concerned with the measurement of performance of solutions to 3DIP problems are supported by this dataset, featuring an extensive number of realistic geometries. This work compared the introduced dataset to the existing benchmarks through a variety of instances and features. We anticipate that this research will include the development of approaches for multi-objective packing problems that consider engineering constraints, such as volume utilisation and surface roughness (Byun and Lee 2005; Mani et al. 2017), and problems that combine packing and scheduling constraints such as Bin Packing problems with heterogeneous containers.

In particular, we anticipate that this paper will help address the AM workflow as a construction problem (Denyer, Tranfield, and Van Aken 2008; van Aken, Chandrasekaran, and Halman 2016). Here, the proposed taxonomy and benchmark could provide important linkages between formal theory, which expresses the relationships among theoretic constructs in a generalised way (Whetten 1989) and substantive theory formulated for a bounded context and limited empirical setting (Glaser and Strauss 1967).

On a practical note, it is expected that the incorporation of external production planning and manufacturing execution data, for example originating from ERP systems or sensors located in the manufacturing environment, will enrich 3DIP in the future. This implies that such problems may be addressable on the basis of big data methodologies requiring an integrated data infrastructure and special knowledge. This provides an exciting opportunity for research towards concurrent optimisation and data science applications, but will inevitably create additional challenges.

\section{Acknowledgement}

The authors are grateful to Rodrigo L. Pinheiro - Automated Scheduling, Optimization and Planning (ASAP) Research Group, School of Computer Science, University of Nottingham - for his work in the implementation of the XML generation module for the dataset.

\section{Funding}

This work was supported by the CNPq (Brazilian Council for Research and Development), grant number 248602/2013-6. 


\section{References}

Achillas, Charisios, Dimitrios Tzetzis, and Maria Olga Raimondo. 2017. "Alternative production strategies based on the comparison of additive and traditional manufacturing technologies." International Journal of Production Research 55 (12): 3497-3509.

Allen, Sam, Edmund K Burke, Matthew Hyde, and Graham Kendall. 2009. "Evolving reusable 3d packing heuristics with genetic programming." In Proceedings of the 11th Annual conference on Genetic and evolutionary computation, 931-938. ACM.

Araújo, Luiz Jonatã Pires, Ender Özcan, Jason Atkin, Martin Baumers, Christopher Tuck, and Richard Hague. 2015. "Toward better build volume packing in additive manufacturing: classification of existing problems and benchmarks." .

Araújo, Luiz Jonatã Pires, Ender Özcan, Jason AD Atkin, Martin Baumers, Chris Tuck, and Richard Hague. 2016. "A part complexity measurement method supporting 3D Printing." .

Araújo, Luiz Jonatã Pires, and Plácido Pinheiro. 2010. "Combining Heuristics Backtracking and Genetic Algorithm to Solve the Container Loading Problem with Weight Distribution." In Soft Computing Models in Industrial and Environmental Applications, 95-102. Springer.

Baumers, Martin, Luca Beltrametti, Angelo Gasparre, and Richard Hague. 2017. "Informing additive manufacturing technology adoption: total cost and the impact of capacity utilisation." International Journal of Production Research 1-14.

Baumers, Martin, Chris Tuck, Ricky Wildman, Ian Ashcroft, and Richard Hague. 2016. "Shape complexity and process energy consumption in electron beam melting: A case of something for nothing in additive manufacturing?" Journal of Industrial Ecology .

Baumers, Martin, Chris Tuck, Ricky Wildman, Ian Ashcroft, Emma Rosamond, and Richard Hague. 2013. "Transparency built-in." Journal of Industrial Ecology 17 (3): 418-431.

Beasley, John E. 1990. "OR-Library: distributing test problems by electronic mail." Journal of the operational research society 41 (11): 1069-1072.

Bischoff, EE. 2006. "Three-dimensional packing of items with limited load bearing strength." European Journal of Operational Research 168 (3): 952-966.

Boccia, Maurizio, Serena Di Muro, Francesco Mosca, Antonio Sforza, and Claudio Sterle. 2011. "A fast heuristic for a three-dimensional non-convex domain loading problem." 4 OR 9 (1): 83-101.

Burke, E. K., M. Gendreau, M. Hyde, G. Kendall, G. Ochoa, E. Özcan, and R. Qu. 2013. "Hyper-heuristics: A Survey of the State of the Art." Journal of the Operational Research Society 64 (12): 1695-1724.

Byun, Hong-Seok, and Kwan H Lee. 2005. "Determination of the optimal part orientation in layered manufacturing using a genetic algorithm." International journal of production research 43 (13): 27092724 .

Canellidis, V, J Giannatsis, and V Dedoussis. 2009. "Genetic-algorithm-based multi-objective optimization of the build orientation in stereolithography." The International Journal of Advanced Manufacturing Technology 45 (7-8): 714-730.

Canellidis, V, J Giannatsis, and V Dedoussis. 2010. "Effective nesting of Layer Manufacturing fabricated parts using a Genetic Algorithm and a bottom-left ray casting procedure." In Industrial Engineering and Engineering Management (IEEM), 2010 IEEE International Conference on, 547-551. IEEE.

Canellidis, Vassilios, Vassilis Dedoussis, N Mantzouratos, and S Sofianopoulou. 2006. "Pre-processing methodology for optimizing stereolithography apparatus build performance." Computers in industry 57 (5): 424-436.

Canellidis, Vassilios, John Giannatsis, and Vassilis Dedoussis. 2013. "Efficient parts nesting schemes for improving stereolithography utilization." Computer-Aided Design 45 (5): 875-886.

Chernov, N, Yu Stoyan, and Tatiana Romanova. 2010. "Mathematical model and efficient algorithms for object packing problem." Computational Geometry 43 (5): 535-553.

Davies, A Paul, and Eberhard E Bischoff. 1999. "Weight distribution considerations in container loading." European Journal of Operational Research 114 (3): 509-527.

de Mul, Jos. 2016. "Possible Printings: On 3D Printing, Database Ontology, and Open (Meta) Design." In $3 D$ Printing, 87-98. Springer.

Denyer, David, David Tranfield, and Joan Ernst Van Aken. 2008. "Developing design propositions through research synthesis." Organization studies 29 (3): 393-413.

Dyckhoff, Harald. 1990. "A typology of cutting and packing problems." European Journal of Operational 
Research 44 (2): 145-159.

Egeblad, Jens. 2009. "Placement of two-and three-dimensional irregular shapes for inertia moment and balance." International Transactions in Operational Research 16 (6): 789-807.

Egeblad, Jens, Benny K Nielsen, and Marcus Brazil. 2009. "Translational packing of arbitrary polytopes." Computational Geometry 42 (4): 269-288.

Egeblad, Jens, Benny K Nielsen, and Allan Odgaard. 2007. "Fast neighborhood search for two-and threedimensional nesting problems." European Journal of Operational Research 183 (3): 1249-1266.

Egeblad, Jens, and David Pisinger. 2009. "Heuristic approaches for the two-and three-dimensional knapsack packing problem." Computers $\&$ Operations Research 36 (4): 1026-1049.

Eisenbrand, Friedrich, Stefan Funke, Andreas Karrenbauer, Joachim Reichel, and Elmar Schömer. 2005. "Packing a trunk: now with a twist!" In Proceedings of the 2005 ACM symposium on Solid and physical modeling, 197-206. ACM.

Gardan, Julien. 2016. "Additive manufacturing technologies: state of the art and trends." International Journal of Production Research 54 (10): 3118-3132.

Garey, Michael R., and David S. Johnson. 1979. Computers and Intractability: A Guide to the Theory of NP-Completeness. New York, NY, USA: W. H. Freeman \& Co.

Gell-Mann, Murray. 2002. "What is complexity?" In Complexity and industrial clusters, 13-24. Springer.

Gibson, Ian, David Rosen, and Brent Stucker. 2014. Additive manufacturing technologies: 3D printing, rapid prototyping, and direct digital manufacturing. Springer.

Glaser, Barney, and Anselm Strauss. 1967. "Grounded theory: The discovery of grounded theory." Sociology The Journal Of The British Sociological Association 12: 27-49.

Gogate, AS, and SS Pande. 2008. "Intelligent layout planning for rapid prototyping." International Journal of Production Research 46 (20): 5607-5631.

Hague, Richard, I Campbell, and Phill Dickens. 2003. "Implications on design of rapid manufacturing." Proceedings of the Institution of Mechanical Engineers, Part C: Journal of Mechanical Engineering Science 217 (1): 25-30.

Hague, Richard, Saeed Mansour, and Naguib Saleh. 2004. "Material and design considerations for rapid manufacturing." International Journal of Production Research 42 (22): 4691-4708.

Hemminki, J, T Leipala, and O Nevalainen. 1998. "On-line packing with boxes of different sizes." International journal of production research 36 (8): 2225-2245.

Hur, Sung-Min, Kyung-Hyun Choi, Seok-Hee Lee, and Pok-Keun Chang. 2001. "Determination of fabricating orientation and packing in SLS process." Journal of Materials Processing Technology 112 (2): 236-243.

Ikonen, Ilkka, William E Biles, Anup Kumar, John C Wissel, and Rammohan K Ragade. 1997. "A Genetic Algorithm for packing three-dimensional non-convex objects having cavities and holes." In ICGA, 591598. Citeseer.

Iori, Manuel, and Silvano Martello. 2010. "Routing problems with loading constraints." Top 18 (1): 4-27.

Jacobs, Paul Francis. 1992. Rapid prototyping 8 manufacturing: fundamentals of stereolithography. Society of Manufacturing Engineers.

Joseph, J, O Sharif, A Kumar, S Gadkari, and A Mohan. 2014. "Using big data for machine learning analytics in manufacturing." Tata Consultancy Services Limited White Paper .

Kagermann, Henning, Wolf-Dieter Lukas, and Wolfgang Wahlster. 2011. "Industrie 4.0: Mit dem Internet der Dinge auf dem Weg zur 4. industriellen Revolution." VDI nachrichten 13: 11.

Karabulut, Korhan, and Mustafa Murat İnceoğlu. 2004. "A hybrid genetic algorithm for packing in 3d with deepest bottom left with fill method." In International Conference on Advances in Information Systems, 441-450. Springer.

Kotthoff, Lars. 2014. "Algorithm selection for combinatorial search problems: A survey." Ai Magazine 35 (3): 48-60.

Lawler, Eugene L, Jan Karel Lenstra, Alexander HG Rinnooy Kan, and David B Shmoys. 1993. "Sequencing and scheduling: Algorithms and complexity." Handbooks in operations research and management science 4: $445-522$.

Lee, Yusin, Chung Fang, You-Ren Tsou, Li-Sin Lu, and Cheng-Tao Yang. 2009. "A packing algorithm for three-dimensional convex particles." Granular Matter 11 (5): 307-315.

Liu, Xiao, Jia-min Liu, An-xi Cao, et al. 2015. "HAPE3Da new constructive algorithm for the 3D irregular packing problem." Frontiers of Information Technology 8 Electronic Engineering 16 (5): 380-390.

Lutters, E, D ten Dam, and T Faneker. 2012. "3D Nesting of Complex Shapes." Procedia CIRP 3: 26-31. 
Mani, Mahesh, Brandon M Lane, M Alkan Donmez, Shaw C Feng, and Shawn P Moylan. 2017. "A review on measurement science needs for real-time control of additive manufacturing metal powder bed fusion processes." International Journal of Production Research 55 (5): 1400-1418.

Nyaluke, Adriano, Bahaa Nasser, Herman R Leep, and Hamid R Parsaei. 1996. "Rapid prototyping work space optimization." Computers \& industrial engineering 31 (1-2): 103-106.

Pisinger, David. 2002. "Heuristics for the container loading problem." European journal of operational research 141 (2): 382-392.

Psarra, Sophia, and Tadeusz Grajewski. 2001. "Describing shape and shape complexity using local properties." In Proceedings 3rd International Space Syntax Symposium, 28-1. Citeseer.

Ravindran, Ashwin. 2003. "An Octree Based Genetic Algorithm for Three-dimensional Packing of Irregular Parts." PhD diss., University of Cincinnati.

Ross, Peter. 2005. "Hyper-heuristics." In Search methodologies, 529-556. Springer.

Ruffo, M, and Richard Hague. 2007. "Cost estimation for rapid manufacturing - simultaneous production of mixed components using laser sintering." Proceedings of the Institution of Mechanical Engineers, Part B: Journal of Engineering Manufacture 221 (11): 1585-1591.

Scheithauer, G, Yu G Stoyan, and T Ye Romanova. 2005. "Mathematical modeling of interactions of primary geometric 3D objects." Cybernetics and Systems Analysis 41 (3): 332-342.

Schmenner, Roger W, and Morgan L Swink. 1998. "On theory in operations management." Journal of operations management 17 (1): 97-113.

Schwab, Klaus. 2017. The fourth industrial revolution. Crown Business.

Slansky, Dick. 2008. "Integrating PLM with the Shop Floor: Validating As-Built to As-Designed." $A R C$ Advisory Group .

Spies, Klaus. 1957. "Die Zwischenformen beim Gesenkschmieden und ihre Herstellung durch Formwalzen." PhD diss., Springer-Verlag; Düsseldorf.

Stafford, D Shane, and Thomas L Jackson. 2010. "Using level sets for creating virtual random packs of non-spherical convex shapes." Journal of Computational Physics 229 (9): 3295-3315.

Stoyan, YG, M Gil, AV Pankratov, and G Scheithauer. 2004. "Packing non-convex polytopes into a parallelepiped." Preprint MATH-NM-06-2004: Technische Universität of Dresden .

Stoyan, YG, NI Gil, G Scheithauer, A Pankratov, and I Magdalina. 2005. "Packing of convex polytopes into a parallelepiped." Optimization 54 (2): 215-235.

Tavakkoli-Moghaddam, Reza, G Moslehi, M Vasei, and Amir Azaron. 2005. "Optimal scheduling for a single machine to minimize the sum of maximum earliness and tardiness considering idle insert." Applied Mathematics and Computation 167 (2): 1430-1450.

Tuck, Christopher John, Richard JM Hague, Massimiliano Ruffo, Michelle Ransley, and P Adams. 2008. "Rapid manufacturing facilitated customization." International Journal of Computer Integrated Manufacturing 21 (3): 245-258.

van Aken, Joan, Aravind Chandrasekaran, and Joop Halman. 2016. "Conducting and publishing design science research: Inaugural essay of the design science department of the Journal of Operations Management." Journal of Operations Management 47: 1-8.

Verkhoturov, Mikhail, Alexander Petunin, Galina Verkhoturova, Konstantin Danilov, and Dmitry Kurennov. 2016. "The 3D Object Packing Problem into a Parallelepiped Container Based on Discrete-Logical Representation." IFAC-PapersOnLine 49 (12): 1-5.

Vokurka, Robert J, and Scott W O'Leary-Kelly. 2000. "A review of empirical research on manufacturing flexibility." Journal of operations management 18 (4): 485-501.

Wäscher, Gerhard, Heike Haußner, and Holger Schumann. 2007. "An improved typology of cutting and packing problems." European Journal of Operational Research 183 (3): 1109-1130.

Weller, Christian, Robin Kleer, and Frank T Piller. 2015. "Economic implications of 3D printing: Market structure models in light of additive manufacturing revisited." International Journal of Production Economics 164: 43-56.

Whetten, David A. 1989. "What constitutes a theoretical contribution?" Academy of management review 14 (4): 490-495.

Wu, Shuohao, Michael Kay, Russell King, Anita Vila-Parrish, and Donald Warsing. 2014. "Multi-objective Optimization of 3D Packing Problem in Additive Manufacturing." . 